\title{
CLINICAL RESEARCH DURING COVID-19 SPREAD: MANAGING AN EMERGENCY WITHIN A PANDEMIC OUTBREAK
}

\author{
C. Cagnazzo ${ }^{1,2}$, S. Testoni ${ }^{3}$, R. Cenna ${ }^{2}$, S. Stabile ${ }^{4}$, E. Tirtei ${ }^{1,2}$, N. Bertorello ${ }^{2}$, \\ F. Fagioli ${ }^{1,2}$ \\ ${ }^{1}$ Department of Public Health and Pediatrics, University of Turin, Turin, Italy \\ 2 Paediatric Haematology-Oncology Department, Regina Margherita Children's Hospital, A.O.U. Città della Salute di \\ Torino, Turin, Italy \\ ${ }^{3}$ IRCCS Istituto Scientifico Romagnolo per lo Studio dei Tumori (IRST) Dino Amadori, Meldola, Italy \\ ${ }^{4}$ Niguarda Cancer Center, Grande Ospedale Metropolitano Niguarda, Milano, Italy
}

\section{CORRESPONDING AUTHOR:}

\section{Celeste Cagnazzo}

Department of Public Health and Pediatrics University of Turin

S.C. Oncoematologia Pediatrica

AOU Città della Salute e della Scienza

Presidio Ospedaliero Infantile Regina Margherita

piazza Polonia 94

10126 Turin, Italy

E-mail: celeste.cagnazzo@unito.it

ORCID: 0000-0003-4055-3139

Doi: $10.48286 / a r o .2021 .02$

History

Received: Oct 1, 2020

Accepted: Oct 29, 2020

Published: Mar 1, 2021

\section{KEY WORDS}

Clinical trials; Covid-19; pandemic; disruption; recommendations.

\section{IMPACT STATEMENT}

The paper provides an overview of the actions taken to preserve clinical research during the pandemic, some of which it would be important to maintain in the future.

pandemic posed an unprecedented challenge for healthcare and research systems, facing the worst crisis in the last 50 years (2).

In the early phase of the pandemic outbreak, Italy was the most affected European country: the first forced 
to a profound re-organization of the healthcare system, to not only effectively handle the pandemic but also keep the routine patient management and care. In this scenario, the research system had to provide scientifically useful and rapid answers, under an unprecedented media pressure.

\section{HEALTHCARE HOSTAGE OF THE COVID-19}

Providing care to patients has been extremely challenging, especially for oncologists.

Cancer patients infected with COVID-19 coronavirus have a 3.5 times increased risk of requiring admission in an intensive care unit (3); moreover, as most of adult Cancer Department resources are used to respond to the health emergency, patients are often treated in centres already suffering, due to the pandemic, from limited resources and instruments (4). In dedicated cancer centres, the general policy was to attempt to stay COVID-19 free, to ensure that enough clinical and intensive - care capacity could be reserved for critical cancer situations (5). An impossible goal for non-dedicated cancer institutions, to the point that international cancer societies decided to spread out priority driven guidelines for the management of onco-hematological patients during the emergency period (6).

\section{READJUSTING CLINICAL TRIALS}

The pandemic has caused a massive disruption in research worldwide; laboratories have closed, communications shut down, conferences cancelled and thousands of clinical trials (around 80\% of non-COVID-19 ones) temporarily or permanently suspended. Furthermore, many researchers have been transferred, especially in the first months of the emergency, from clinical trials activity to operating in emergency and/or COVID-19 dedicated units (7).

A marked decline in screening and patient accrual has been detected (8), although in many cases only new recruitments were suspended, while already included patients continued to receive treatment thanks to alternative plans, jointly implemented by founders, institutions and regulatory authorities. This profound disruption has also affected the field of paediatric cancer, the early-phase clinical research above all (9).
During COVID breakdown, Pharmaceutical Industries and Sponsors made efforts to accelerate trial innovations providing digital tools thus allowing virtualization of certain processes to protect patient safety and trial integrity, also with support from regulatory guidelines (10). Simultaneously, Regulatory Agencies, first of all the Italian "Agenzia Italiana del Farmaco (AIFA)", have implemented extrAROdinary measures to guide stakeholders in ensuring patient care and maintaining good data quality (11-13).

Indeed, we can pinpoint the main goals of these regulatory guidelines in clinical research being: the safeguard of patient safety, guaranteeing therapeutic continuity and the work ship of data integrity and consistency. Actual operating instructions are widely spread also with the help of scientific societies (14). New trials regarding COVID-19 infection were fast tracked, existing inefficiencies were promptly identified and streamlined, Good Clinical Practices (GCP) applied in a less conservative way, demonstrating that a reasonable balance between patient safety, regulatory burden, scientific quality and integrity may not be a utopia.

Patients remain central in any decision at all times from clinical to research activities, also during the adjustment of ongoing clinical trials. This centrality is the driving force behind various guidelines, leading especially in Italy, to the unhinging of rules that seem like engraved in stone.

Below, we highlight five innovation key points introduced by the guidelines for the management of clinical studies in the emergency period. Five notable reflections, perhaps worthy of being taken into consideration even in non-emergency times.

\section{Telemedicine and activities outside the experimental site}

Travel restrictions adopted during the pandemic led to delays and in some cases the impossibility for many patients to reach the trial sites at scheduled visits and laboratory or instrumental tests. In order to minimize the risk for patients to withdraw from treatment, regulatory authorities granted the possibility to perform blood tests, imaging or other diagnostic tests at the nearest local facility, provided it be certified as per national requirements.

This experience may be continued and integrated into clinical trial procedures, especially for the benefit of patients living far from specialized centres, at least for procedures not correlated to the primary endpoint of the study. Obviously if this practice becomes 
routine, valid mechanisms should be established to track and reimburse these extra institutional procedures. In addition, the staff of the peripheral centres should be adequately trained, and a clear division of responsibilities must be put in place under the supervision of the team, this being an objective easily achieved by strengthening the research networks (15). Efforts to protect the patient have gone so far as to enable direct dispatch of oral medication from the hospital pharmacy to the patient's home, another practice that, after careful planning and adequate workforce, may become standard procedure.

The need for physical distancing to protect patients and research staff also motivated Countries to rapidly implement telemedicine programs (16). Telemedicine has already been around for 20 years, however before the pandemic it was mostly underused and in many cases hampered by administrative and/or bureaucratic barriers, such as the need for costly nationwide standardized payment policies, that ultimately prevented this approach from being incorporated into clinical practice or clinical studies (17).

Having said that, many study-specific activities can be potentially provided through electronic tools: medical history collection, evaluation of quality of life, informed consent discussion and signature, re-consent and follow up visits are just some examples.

\section{Decentralization and remote oversight}

Another consequence of the restrictions imposed by the pandemic concerned the impossibility of carrying out on-site monitoring visits by CRA or Sponsor delegates, forcing replacement with alternative forms of oversight and monitoring.

Despite the fact that some forms of monitoring and especially of source data verification (e.g., use of Skype or Zoom) have currently been prohibited in many European Countries by local data protection policies, alternative ways such as mixed and riskbased systems, should not be excluded and could make way for large economic savings, all the while guaranteeing patient safety. This is especially true for studies promoted by non-profit organizations (18). There is no doubt that this evolution cannot occur without a general technological advancement of healthcare, especially in Italy, starting from electronic medical records, that are still far from being successfully or uniformly implemented.

Furthermore, remote work should become standard practice at least partially for audits and inspections, albeit maintaining certain activities that cannot be deferred on site.

\section{A single ethical evaluation}

The process adopted to quick start COVID-19 trials in Italy, which requires the approval by the Agenzia Italiana del Farmaco (AIFA) and that of only one Ethics Committee (EC), instead of every EC of each experimental centre involved, has significantly shrinked the timeline for authorization (14.1 \pm 9.8 days rather than a mean of about 150 days). This was the biggest novelty for Italy, for a long time accustomed to a multitude of Ethics Committees and a redundancy of start-up procedures, often suffocating studies.

Considering the success of this approval process during the pandemic, it would be desirable to maintain it, also considering that this would finally bring us in line with the provisions of the European Regulation 536/2014 already fully implemented by other European Countries like Spain, and ultimately slashing costs of submission. Moreover, this could be applied to different types of clinical research, observational studies included.

Some concerns regarding this approach regard the possible overload for the Ethics Committee and the risk that a single ethical opinion might reduce the strictness of the evaluations. This being said, the current number of Ethics Committees existing in Italy (around ninety) and the number required by the Law 3/2018 should allow a not so onerous distribution of the authorization procedures.

Intermediate solutions could be evaluated, such as the establishment of a few highly specialized committees in the various areas of research, or in specific pathologies, which could be called upon depending on their expertise and on the subject of the experimentation, all the while guaranteeing that a national opinion is expressed.

\section{Bureaucracy give way to science}

The administrative burden of clinical research is a problem that has been alarming stakeholders (18) and contract and budget negotiations have been identified as time-consuming procedures interfering with study participation, to the point that the American Society of Clinical Oncology recommended the adoption of standardized contract templates (19). Although in Italy the National Coordination Centre of Ethics Committees recently released a standard contract template for profit interventional studies, the negotiation process is still excessively centre-dependent and delayed by additional procedures imposed by the individual institutions and/ or ethical committees. In addition, there are still ongoing redundant procedures requiring periodic 
collection of identical documents that could easily be shared between study promoters (e.g., curricula, trainings, certifications) and the reluctance to adopt simplifications that could save a lot of time and energy, trivially the use of the electronic signature.

On this subject, many exceptions have been granted during the pandemic thus enabling us to witness in Italy the kick-start of an academic study with the involvement of 600 centres in just 3 weeks. Note this was a prestigious study, evaluated by AIFA. Finally, we ought to necessarily stop and consider how much work we have done so far was really useful in order for bureaucracy to give way to science once and for all.

\section{No chance for research without professionals}

The emergency period has emphasized how professionals dedicated to the management of the clinical trials and data collection, such as the Study Coordinator and Study Nurses, play a crucial role for the success of a clinical study, particularly in support of Investigators.

The current legislation requires their mandatory presence for phase 1 centres, and the Law 3/2018 imposes that "clinical trials of medicines make use of specific professionalism in the field of data management and research coordination". However, these professional figures are substantially under-represented; adding to, a considerable heterogeneity in terms of education backgrounds, training and job descriptions, there is the of contractual stabilization and the lack of professional recognition at an institutional level $(20,21)$.

Nevertheless, there is ample evidence in literature that the presence of research infrastructures increases the performance of the centre and these are now mandatory, also given the whirling increase in the complexity of the research (22-24).

During the pandemic there was a deep gap between structures with strong infrastructures, which managed to keep their research projects going and even promote new ones, also thanks to the possibility of implementing smart working (25), and small research centres which, having no adequate staff, were forced to stop clinical research activity all together.

Especially in view of at full application of European legislation, which will greatly increase the complexity in the management of studies for non-profit organizations, the stabilization of these resources can no longer be postponed (26).

\section{PREPARING FOR THE FUTURE}

The COVID-19 pandemic has caused an unparalleled global emergency, but at the same time it has triggered a profound analysis of ethics and research organization. Above all, it has allowed to make a way for a new vision of clinical research.

Clinical trials are an essential tool for scientific progress but COVID-19 has exposed aspects regarding their design and conduct that could be improved and simplified. Most trial aspects could be streamlined and modernized, and bureaucracy lightened without dramatic consequences at the expenses of research quality and consistency.

The innovations introduced during the pandemic by regulatory authorities have proved so successful that many stakeholders are clamouring to keep them even when the pandemic will be finally over (27-30).

Primarily Italy, which has always been pointed out as a slow Country with excessively cumbersome bureaucracy, through the joint work of Institution, regulatory authorities and stakeholders could recover its prestigious place in the world of research. The important lessons learned during the pandemic must not disappear at the end of the emergency.

\section{ETHICS}

\section{Fundings}

There were no institutional or private funding for this article.

\section{Conflict of interests}

The authors have declared no conflict of interests.

\section{Availability of data and material}

The data underlying this article can be shared just before a reasonable request to the corresponding author.

\section{Authors' contribution}

CC: conceptualization; CC, ET: methodology; CC, FF, ST, SS: investigation; CC, RC: writing - original draft preparation; ST, SS, NB, ET: writing - review \& editing; FF: supervision; CC: project administration.

\section{Ethical approval}

N/A 


\section{REFERENCES}

1. World Health Organization. Coronavirus disease (COVID-19) situation report. 2020. Available at: https://www.who.int/emergencies/diseases/ novel-coronavirus-2019/situation-reports. Accessed: 18/01/2021.

2. Emanuel EJ, Persad G, Upshur R, et al. Fair Allocation of Scarce Medical Resources in the Time of Covid-19. N Engl J Med 2020;382(21):2049-55.

3. Liang W, Guan W, Chen R, et al. Cancer patients in SARS-CoV-2 infection: a nationwide analysis in China. Lancet Oncol 2020;21(3):335-7.

4. Ueda M, Martins R, Hendrie PC, et al. Managing Cancer Care During the COVID-19 Pandemic: Agility and Collaboration Toward a Common Goal. J Natl Compr Canc Netw 2020:1-4.

5. van de Haar J, Hoes LR, Coles CE, et al. Caring for patients with cancer in the COVID-19 era. Nat Med 2020;26(5):665-71.

6. Tagliamento M, Lambertini M, Genova C, et al. Call for ensuring cancer care continuity during COVID-19 pandemic. ESMO Open 2020;5(3).

7. van Dorn A. COVID-19 and readjusting clinical trials. Lancet 2020;396(10250):523-4.

8. Ong $\mathrm{MBH}$. Doroshow: $\mathrm{NCl}$ to accrue patients for COVID-19 longitudinal cohort. 2020.

9. Rubio-San-Simón A, André N, Cefalo MG, et al. Impact of COVID-19 in paediatric early-phase cancer clinical trials in Europe: A report from the Innovative Therapies for Children with Cancer (ITCC) consortium. Eur J Cancer 2020;141:82-91.

10. Xue JZ, Smietana K, Poda P, et al. Clinical trial recovery from COVID-19 disruption. Nat Rev Drug Discov 2020;19(10):662-663.

11. European Medicines Agency. Guidance On The Management Of Clinical Trials During The Covid-19 (Coronavirus) Pandemic. 2020. Available at: https://ec.europa.eu/health/sites/health/files/ files/eudralex/vol- 10/guidanceclinicaltrials_covid19_en.pdf. Accessed: 18/01/2021.

12. Agenzia Italiana del Farmaco. Gestione degli studi clinici in Italia in corso di emergenza COVID-19 (coronavirus disease 19). 2020. Available at: https://www.aifa.gov.it/-/gestione-degli-studi-clinici-in-italia-in-corso-di-emergenza-covid-19-aggiornamento-del-17-settembre-2020-. Accessed: 18/01/2021.

13. Food and Drug Administration . Guidance on Conduct of Clinical Trials of Medical Products during COVID-19 Public Health Emergency. 2020. Available at: https://www.fda.gov/regulatory-information/search-fda-guidance-documents/fda-guidance-conduct-clinical-trials-medical-products-during-covid-19-publichealth-emergency. Accessed 25/11/2021.

14. Pinto C, Cagnazzo C. Indications regarding the management of interventional clinical trials with drugs during the current COVID-19 emergency in Italy. ESMO Open 2020;5(2).

15. Doherty GJ, Goksu M, de Paula BHR. Rethinking cancer clinical trials for COVID-19 and beyond. Nat Cancer 2020:1-5.

16. Hollander JE, Carr BG. Virtually Perfect? Telemedicine for Covid-19. N Engl J Med 2020;382(18): 1679-81.

17. Men J. Lack of reimbursement barrier to telehealth adoption. 2015. Available at:https://www. ajmc.com/view/lack-of-reimbursement-barrier-to-telehealth-adoption. Accessed 18/01/2021.

18. Uren SC, Kirkman MB, Dalton BS, et al. Reducing clinical trial monitoring resource allocation and costs through remote access to electronic medical records. J Oncol Pract 2013;9(1): e13-16.

19. Thompson MA, Hurley PA, Faller B, et al. Challenges With Research Contract Negotiations in Community-Based Cancer Research. J Oncol Pract 2016;12(6): e626-32.

20. Cagnazzo C, Testoni S, Guarrera AS, et al. [Clinical research coordinators: a crucial resource.]. Recenti Prog Med 2019;110(2):65-7.

21. Martucci M, Guarrera A, Valente D, et al. Clinical research nurse in Italian centers: a mandatory figure?. Recenti Prog Med 2020;111(9):535-8.

22. Emanuel EJ, Schnipper LE, Kamin DY, et al. The costs of conducting clinical research. J Clin Oncol 2003;21(22):4145-50.

23. Davis AM, Hull SC, Grady C, et al. The invisible hand in clinical research: the study coordinator's critical role in human subjects protection. J Law Med Ethics 2002; 30(3):411-9.

24. Street A, Strong J, Karp S. Improving patient recruitment to multicentre clinical trials: the case for employing a data manager in a district general hospital-based oncology centre. Clin Oncol (R Coll Radiol) 2001;13(1):38-43.

25. Cagnazzo C, Franchina V, Maggiora $P$, et al. Revolutionizing clinical trial units in the pandemic era. Ann Oncol 2020; 31 (Supll 4). 
26. Cagnazzo C, Guarrera A, Cenna R, et al. Clinical research: enough players to get out there?. Recenti Prog Med 2019;110(6):285-91.

27. Cagnazzo C, Fagioli F. Bureaucracy gives way to science. What good the pandemic has left.. Recenti Prog Med 2020;111(10):565-7.

28. Lorusso D, Ray-Coquard I, Oaknin A, et al. Clinical research disruption in the post-COVID-19 era: will the pandemic lead to change? ESMO Open 2020;5(5).
29. Cagnazzo C, Besse MG, Manfellotto D, et al. Lessons learned from CoViD-19 for clinical research operations in Italy: what have we learned and what can we apply beyond? Tumori 2020. 9.

30. TransCelerate Biopharma INC. Beyond COVID-19: Modernizing Clinical Trial Conduct. 2020. Available at: https://www.transceleratebiopharmainc. com/wp-content/uploads/2020/08/TransCelerate_Beyond-COVID19_Modernizing-Clinical-Trial-Conduct July-2020.pdf. Accessed: 18/01/2021. 